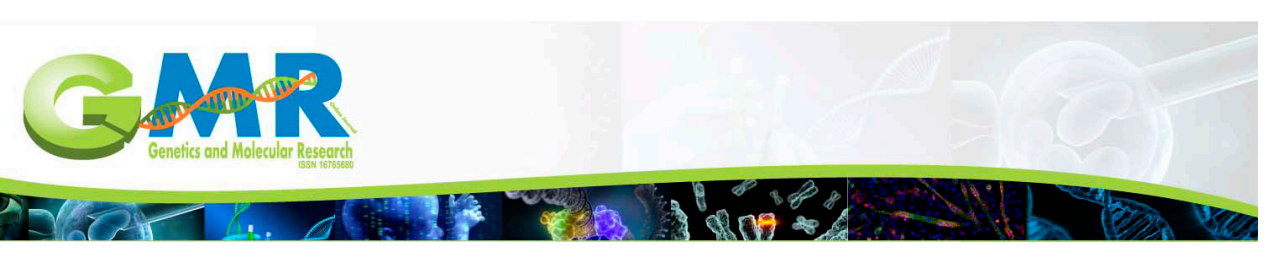

$\underline{\text { Case report }}$

\title{
Identification of a novel SLC45A2 mutation in albinism by targeted next-generation sequencing
}

\author{
J.J. Xue ${ }^{1}$, J.F. Xue ${ }^{2}$, H.Q. Xue ${ }^{1}$, Y.Y. Guo ${ }^{3}$, Y. Liu ${ }^{3}$ and N. Ouyang ${ }^{4}$ \\ ${ }^{1}$ Children's Hospital of Shanxi, Women Health Center of Shanxi, Taiyuan, \\ Shanxi, China \\ ${ }^{2}$ Yuncheng Central Hospital, Yuncheng, Shanxi, China \\ ${ }^{3}$ Beijing Genomics Institute at Shenzhen, Shenzhen, China \\ ${ }^{4}$ Women Health Center of Pingxiang, Pingxiang, Jiangxi, China \\ Corresponding author: J.J. Xue \\ E-mail: xuejinjiesxsfy@126.com
}

Genet. Mol. Res. 15 (3): gmr.15038743

Received April 28, 2016

Accepted June 22, 2016

Published September 19, 2016

DOI http://dx.doi.org/10.4238/gmr.15038743

Copyright $(2016$ The Authors. This is an open-access article distributed under the terms of the Creative Commons Attribution ShareAlike (CC BY-SA) 4.0 License.

\begin{abstract}
Albinism is a diverse group of hypopigmentary disorders caused by multiple-genetic defects. The genetic diagnosis of patients affected with albinism by Sanger sequencing is often complex, expensive, and time-consuming. In this study, we performed targeted next-generation sequencing to screen for 16 genes in a patient with albinism, and identified 21 genetic variants, including 19 known single nucleotide polymorphisms, one novel missense mutation (c.1456 G>A), and one disease-causing mutation (c.478 $\mathrm{G}>\mathrm{C}$ ). The novel mutation was not observed in 100 controls, and was predicted to be a damaging mutation by SIFT and Polyphen. Thus, we identified a novel mutation
\end{abstract}

Genetics and Molecular Research 15 (3): gmr.15038743 
in SLC45A2 in a Chinese family, expanding the mutational spectrum of albinism. Our results also demonstrate that targeted next-generation sequencing is an effective genetic test for albinism.

Key words: Targeted next-generation sequencing; Albinism; SLC45A2; Novel mutation

\section{INTRODUCTION}

Albinism is a common hereditary disorder characterized by decreased or lack of pigmentation in the hair, skin, and eyes. Based on its clinical phenotypes, albinism has been classified into the following types: ocular albinism, characterized by decreased pigmentation (primarily) in the eyes (Xiao and Zhang, 2009); oculo-cutaneous albinism, characterized by decreased pigmentation in the eyes, hair, and skin; and syndromic albinism (Berrueco et al., 2010). Albinism is a highly heterogeneous genetic disorder with an autosomal recessive, autosomal dominant, or X-linked inheritance pattern. To date, approximately 16 albinismrelated genes (GPR143, TYR, OCA2, TYRP1, SLC45A2, SLC24A5, C10ORF11, HPS1, AP3B1, HPS3, HPS4, HPS5, HPS6, DTNBP1, BLOC1S3, and BLOC1S6) have been identified (Morgan et al., 2006; Badolato et al., 2012; Lowe et al., 2013). Today, patients with albinism are primarily diagnosed by Sanger sequencing of one or more of these candidate genes. This approach may be time-consuming, expensive, and may not always identify the diseasecausing gene. Alternately, targeted next-generation sequencing (NGS) is a rapid, accurate, high-throughput, and relatively low-cost method. In this study, we performed targeted

enrichment and NGS to screen for these 16 genes in a patient with albinism, and identified a novel missense mutation in SLC45A2.

\section{MATERIAL AND METHODS}

This study was approved by the hospital Ethics Committees, and informed consent was obtained from the patient and her parents. The patient, the only daughter of healthy non-consanguineous Chinese parents with an unremarkable family history, was born at term after an uneventful pregnancy, and presented white hair and skin. The patient was clinically diagnosed with albinism at the Department of Dermatology. The girl was referred to us at the age of 9 months (with her parents) for genetic counseling. The girl showed normal psychomotor development and displayed the characteristic features of albinism: white hair, white eyebrows and eyelashes, brown irises, and white skin (Figure 1A). Ophthalmoscopic examination revealed ophthalmologic abnormalities such as reduced retinal pigmentation with visualization of the choroidal blood vessels and foveal hypoplasia.

Genomic DNA was extracted from the peripheral blood leukocytes of the patient and her parents, using the DNeasy Tissue kit (Qiagen, Venlo, Netherlands) according to the manufacturer protocols. A capture array (NimbleGen; Roche, Basel, Switzerland) was designed to capture all coding regions and the intron/exon boundaries of the 16 target genes. DNA target capture, enrichment, and elution were performed according to the manufacturer protocols. The sequencing primer was hybridized and the base was incorporated using an Illumina HiSeq2000 analyzer (Illumina, San Diego, CA, USA) according to the standard sequencing protocols of the manufacturer. Image analyses, error estimation, and base calling

Genetics and Molecular Research 15 (3): gmr.15038743 
were performed using Illumina Pipeline (v.1.3.4). After removing a few unqualified sequences, the clean reads were aligned against the reference human genome from the NCBI database. Single nucleotide polymorphisms (SNPs) were determined using the NCBI dbSNP and HapMap databases, least square mean determination, and the 1000 human genome dataset. Known disease-causing mutations were identified from the Human Gene Mutation Database (HGMD) or from mutations previously reported in the literature.

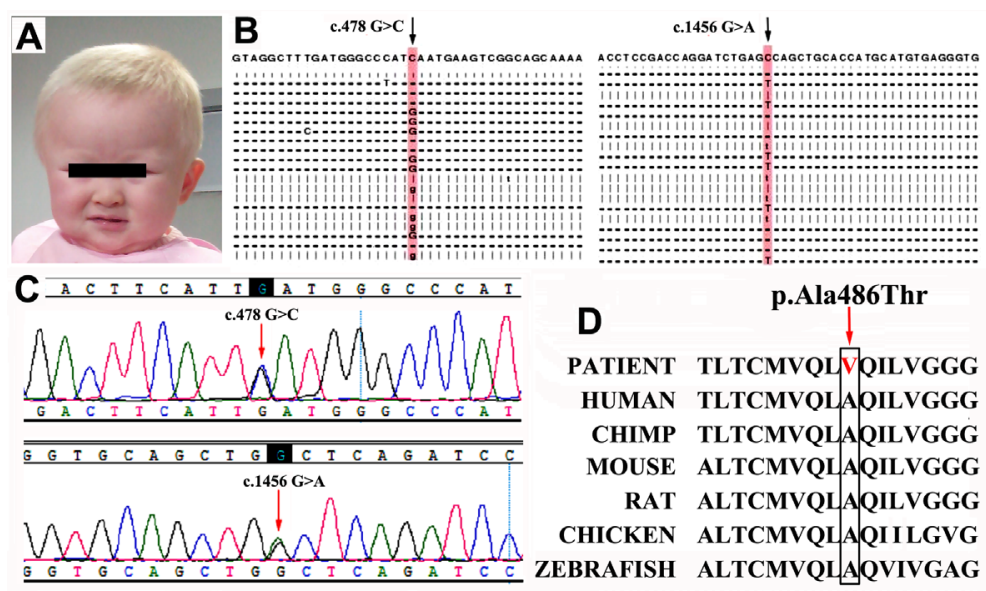

Figure 1. A. Photograph showing the phenotypic characteristics of the proband affected with albinism. B. Targeted next-generation sequencing result of the patient, showing two heterozygous mutations in SLC45A2. C. Sanger sequencing result of the two heterozygous mutations in SLC45A2. D. Evolutionary conservation of amino acid affected by the mutation. The mutant allele is boxed.

The regions containing the novel mutations were amplified by polymerase chain reaction (PCR). Primers designed previously by Rundshagen et al. (2004) were used in this study. The PCR products were sequenced using the ABI Big Dye terminator cycle sequencing kit (Applied Biosystems) according the manufacturer instructions, and run on an ABI 3100 sequencer. The sequencing results were analyzed using DNASTAR ${ }^{\circledR}$.

\section{RESULTS}

Twenty-one gene variants, including 19 known SNPs, one novel missense mutation, and one disease-causing mutation (Figure 1B) were identified in this study. The diseasecausing mutation (c.478 G>C) in SLC45A2 has been previously reported by Wei et al. (2011) and verified by Sanger sequencing (Figure 1C). Additionally, a novel mutation (c.1456 G>A) was identified in SLC45A2. This missense mutation, causing a substitution of the conserved (functionally important) alanine at position 486 with threonine (Figure 1D), has not been reported previously, and was not observed in 100 healthy controls. Sanger sequencing of the family members revealed a heterozygous mutation (c.478 G>C) in $S L C 45 A 2$ in the father and a heterozygous c. $1456 \mathrm{G}>\mathrm{A}$ mutation in the mother. This indicated that the mutations segregate with albinism in the family. Moreover, SIFT and Polyphen prediction demonstrated that the novel mutation (c.1456 G>A) was, in fact, a damaging mutation. These data indicate that this novel mutation may abolish the gene function and cause albinism in this family.

Genetics and Molecular Research 15 (3): gmr.15038743 


\section{DISCUSSION}

The human SLC45A2 (OMIM 606202) gene was mapped to chromosome 5p13.2 and encodes a protein containing 530 amino acids (Fukamachi et al., 2001). SLC45A2 contains 12 putative transmembrane domains and may function as a membrane transporter, associated with tyrosinase processing and intracellular trafficking to the melanosome (Ko et al., 2012). The novel SLC45A2 mutation (c.1456 G>A) located in the transmembrane domain 11 of the coding protein may disrupt the normal maturation of melanosomes. A missense mutation (c.1457 C>T) at this codon has been reported to lead to oculo-cutaneous albinism 4 (Rundshagen et al., 2004), suggesting that the alanine 486 plays a key role in the function of the transmembrane domain and the SLC45A2 protein. The major limitation of this study is that the novel mutation (c.1456 G>A) was only identified in one patient. Therefore, this variation must be scanned in more samples and its pathological mechanism must be analyzed in further studies. In summary, we have identified a novel c.1456 G>A mutation in SLC45A2 in a family with albinism, adding to the repertoire of $S L C 45 A 2$ mutations. Moreover, our results demonstrate that targeted NGS is an effective genetic test for albinism.

\section{Conflicts of interest}

The authors declare no conflict of interest.

\section{ACKNOWLEDGMENTS}

The authors would like to thank the participating family members for their help and support. Research supported by a grant provided by the National Natural Science Foundation of China (\#81200875).

\section{REFERENCES}

Badolato R, Prandini A, Caracciolo S, Colombo F, et al. (2012). Exome sequencing reveals a pallidin mutation in a Hermansky-Pudlak-like primary immunodeficiency syndrome. Blood 119: 3185-3187. http://dx.doi.org/10.1182/ blood-2012-01-404350

Berrueco R, Rives S, Camós M, Toll T, et al. (2010). Syndromic albinism and haemophagocytosis. Br. J. Haematol. 148: 815.http://dx.doi.org/10.1111/j.1365-2141.2009.07885.x

Fukamachi S, Shimada A and Shima A (2001). Mutations in the gene encoding B, a novel transporter protein, reduce melanin content in medaka. Nat. Genet. 28: 381-385. http://dx.doi.org/10.1038/ng584

Ko JM, Yang JA, Jeong SY and Kim HJ (2012). Mutation spectrum of the TYR and SLC45A2 genes in patients with oculocutaneous albinism. Mol. Med. Rep. 5: 943-948.

Lowe GC, Sánchez Guiu I, Chapman O, Rivera J, et al.; UK GAPP collaborative (2013). Microsatellite markers as a rapid approach for autozygosity mapping in Hermansky-Pudlak syndrome: identification of the second HPS7 mutation in a patient presenting late in life. Thromb. Haemost. 109: 766-768. http://dx.doi.org/10.1160/TH12-11-0876

Morgan NV, Pasha S, Johnson CA, Ainsworth JR, et al. (2006). A germline mutation in BLOC1S3/reduced pigmentation causes a novel variant of Hermansky-Pudlak syndrome (HPS8). Am. J. Hum. Genet. 78: 160-166. http://dx.doi. org $/ 10.1086 / 499338$

Rundshagen U, Zühlke C, Opitz S, Schwinger E, et al. (2004). Mutations in the MATP gene in five German patients affected by oculocutaneous albinism type 4. Hum. Mutat. 23: 106-110. http://dx.doi.org/10.1002/humu.10311

Wei A, Yang X, Lian S and Li W (2011). Implementation of an optimized strategy for genetic testing of the Chinese patients with oculocutaneous albinism. J. Dermatol. Sci. 62: 124-127. http://dx.doi.org/10.1016/j.jdermsci.2011.02.009

Xiao X and Zhang Q (2009). Iris hyperpigmentation in a Chinese family with ocular albinism and the GPR143 mutation. Am. J. Med. Genet. A. 149A: 1786-1788. http://dx.doi.org/10.1002/ajmg.a.32818

Genetics and Molecular Research 15 (3): gmr.15038743 